\title{
Research Article \\ Best Proximity Pairs for Upper Semicontinuous Set-Valued Maps in Hyperconvex Metric Spaces
}

\author{
A. Amini-Harandi, ${ }^{1}$ A. P. Farajzadeh ${ }^{2}{ }^{2}$ D. O'Regan, $^{3}$ and R. P. Agarwal ${ }^{4}$ \\ ${ }^{1}$ Department of Mathematics, Faculty of Basic Sciences, University of Shahrekord, \\ Shahrekord 88186-34141, Iran \\ ${ }^{2}$ Department of Mathematics, School of Science, Razi University, Kermanshah 67149, Iran \\ ${ }^{3}$ Department of Mathematics, College of Arts, Social Sciences and Celtic Studies, \\ National University of Ireland, Galway, Ireland \\ ${ }^{4}$ Department of Mathematical Sciences, College of Science, Florida Institute of Technology, \\ Melbourne, FL 32901, USA
}

Correspondence should be addressed to A. Amini-Harandi, aminih_a@yahoo.com

Received 14 July 2008; Accepted 27 October 2008

Recommended by Nan-jing Huang

A best proximity pair for a set-valued map $F: A \multimap B$ with respect to a map $g: A \rightarrow A$ is defined, and new existence theorems of best proximity pairs for upper semicontinuous set-valued maps with respect to a homeomorphism are proved in hyperconvex metric spaces.

Copyright (c) 2008 A. Amini-Harandi et al. This is an open access article distributed under the Creative Commons Attribution License, which permits unrestricted use, distribution, and reproduction in any medium, provided the original work is properly cited.

\section{Introduction and preliminaries}

Let $(M, d)$ be a metric space and let $A$ and $B$ be nonempty subsets of $M$. Let $g: A \rightarrow A$ and let $F: A \multimap B$ be a set-valued map. Now, $(g(a), F(a))$ is called a best proximity pair for $F$ with respect to $g$ if $d(g(a), F(a))=d(A, B)$, where $d(A, B)=\inf \{d(a, b): a \in A, b \in B\}$. Best proximity pair theorems establish conditions under which the problem of minimizing the real-valued function $x \rightarrow d(g(x), F(x))$ has a solution. In the setting of normed linear spaces, the best proximity pair problem has been studied by many authors for $g=I$, see [1-5]. Very recently, Al-Thagafi and Shahzad [1] proved some existence theorems for a finite family of Kakutani set-valued maps in a normed space setting. In the present paper, our aim is to prove new results in hyperconvex metric spaces. In the rest of this section, we recall some definitions and theorems which are used in Section 2.

Let $X$ and $Y$ be topological spaces with $A \subseteq X$ and $B \subseteq Y$. Let $F: X \multimap Y$ be a setvalued map with nonempty values. The image of $A$ under $F$ is the set $F(A)=\bigcup_{x \in A} F(x)$ and the inverse image of $B$ under $F$ is $F^{-}(B)=\{x \in X: F(x) \cap B \neq \varnothing\}$. Now, $F$ is said to be upper semicontinuous, if for each closed set $B \subseteq Y, F^{-}(B)=\{x \in X: F(x) \cap B \neq \varnothing\}$ is closed in $X$. 
A topological space $X$ is said to be contractible if the identity map $I_{X}$ of $X$ is homotopic to a constant map and acyclic if all of its reduced Čech homology groups over the rationals vanish. Note that a contractible space is acyclic. For topological spaces $X$ and $Y$, we define

$$
F \in \mathbb{V}(X, Y) \Longleftrightarrow F: X \multimap Y \text { is an acyclic map; that is, }
$$

$F$ is upper semicontinuous with compact acyclic values.

We denote by $\mathbb{V}_{c}(X, Y)$ the set of all finite composites of maps in $\mathbb{V}(X, Y)$. Let $(M, d)$ be a metric space and let $B(x, r)=\{y \in M: d(x, y) \leq r\}$ denote the closed ball with center $x$ and radius $r$. Let

$$
\operatorname{co}(A)=\bigcap\{B \subseteq M: B \text { be a closed ball in } M \text { such that } A \subseteq B\} .
$$

If $A=\operatorname{co}(A)$, we say that $A$ is admissible subset of $M$. Note that $\operatorname{co}(A)$ is admissible and the intersection of any family of admissible subsets of $M$ is admissible. The following definition of a hyperconvex metric space is due to Aronszajn and Pantichpakdi [6].

Definition 1.1. A metric space $(M, d)$ is said to be a hyperconvex metric space if for any collection of points $x_{\alpha}$ of $M$ and any collection $r_{\alpha}$ of nonnegative real numbers with $d\left(x_{\alpha}, x_{\beta}\right) \leq r_{\alpha}+r_{\beta}$, one has

$$
\bigcap_{\alpha} B\left(x_{\alpha}, r_{\alpha}\right) \neq \varnothing
$$

The simplest examples of hyperconvex spaces are finite dimensional real Banach spaces endowed with the maximum norm. For other examples of hyperconvex metric spaces which are not linear spaces, see [7]. Note that an admissible subset of a hyperconvex metric space is hyperconvex and contractible [8]. Let $A$ be a subset of $M$. The $r$-parallel of $A$ is defined as

$$
A+r=\bigcup_{a \in A} B(a, r)
$$

The following result is due to Sine [9].

Lemma 1.2. The r-parallel sets of an admissible subset of a hyperconvex metric space are also admissible sets.

For $A \subseteq M$, the set $P_{A}(x)=\{a \in A: d(a, x)=d(x, A)\}$ is called the set of best approximations in $A$ to $x \in M$. The map $P_{A}: M \multimap A$ is called the metric projection on $A$. The following lemma is well known. We give its proof for completeness.

Lemma 1.3. Let $A$ be a nonempty, admissible, and compact subset of a hyperconvex metric space $(M, d)$. Then $P_{A} \in \mathbb{V}(M, A)$. 
Proof. Since $A$ is compact, then $P_{A}$ is nonempty. We now show that $P_{A}$ is contractible and so is acyclic. To see this, notice that

$$
P_{A}(x)=A \cap\{a \in M: d(a, x) \leq d(x, A)\}=A \cap B(x, d(x, A)) .
$$

Then $P_{A}(x)$ is admissible (note that $A$ is admissible) and therefore is contractible. Now, we show that $P_{A}$ is upper semicontinuous. Let $C$ be a closed subset of $A, x_{n} \in\left\{x: P_{A}(x) \cap C \neq \varnothing\right\}$, and $x_{n} \rightarrow x_{0}$. Then there exists a sequence $a_{n} \in P_{A}\left(x_{n}\right) \cap C$ such that $d\left(a_{n}, x_{n}\right)=d\left(x_{n}, A\right)$. Since $A$ is compact and $a_{n} \in A$, without loss of generality, we may assume that $a_{n} \rightarrow a$. Thus,

$$
d\left(a, x_{0}\right)=\lim _{n \rightarrow \infty} d\left(a_{n}, x_{n}\right)=\lim _{n \rightarrow \infty} d\left(x_{n}, A\right)=d\left(x_{0}, A\right) .
$$

Therefore, $x_{0} \in\left\{x: P_{A}(x) \cap C \neq \varnothing\right\}$ and the set $\left\{x: P_{A}(x) \cap C \neq \varnothing\right\}$ is closed.

To prove our main result, we need the following fixed point theorem, which is particular form of Theorem 4 in [10].

Theorem 1.4. Let $X$ be a nonempty compact admissible subset of a hyperconvex metric space $(M, d)$ and $F \in \mathbb{V}_{c}(X, X)$. Then $F$ has a fixed point.

Corollary 1.5. Let $X$ be a nonempty compact admissible subset of a hyperconvex metric space $(M, d)$, $g: X \rightarrow X$ a homeomorphism, and $F \in \mathbb{V}_{c}(X, X)$. Then there exists an $x_{0} \in X$ such that $g\left(x_{0}\right) \in$ $F\left(x_{0}\right)$.

Proof. Since $g$ is a homeomorphism, then $g^{-1} \circ F \in \mathbb{V}_{c}(X, X)$. Hence, by Theorem $1.4, g^{-1} \circ F$ has a fixed point, say $x_{0}$. Therefore, $g\left(x_{0}\right) \in F\left(x_{0}\right)$.

\section{Best proximity theorems}

Let $A$ and $B$ be nonempty subsets of $M$. Define

$$
\begin{aligned}
& A^{0}:=\{a \in A: d(a, b)=d(A, B) \text { for some } b \in B\}, \\
& B^{0}:=\{b \in B: d(a, b)=d(A, B) \text { for some } a \in A\} .
\end{aligned}
$$

Notice that $A^{0}$ is nonempty if and only if $B^{0}$ is nonempty.

Theorem 2.1. Let $A$ and $B$ be nonempty subsets of $M$. Then the following statement holds.

(i) If $A^{0}$ and $B$ are admissible, then $B^{0}$ is admissible.

(ii) If $A^{0}$ and $B$ are compact, then $B^{0}$ is compact. 
Proof. To prove (a), notice that

$$
\begin{aligned}
B^{0} & :=\{b \in B: d(a, b)=d(A, B) \text { for some } a \in A\} \\
& =\left\{b \in B: d(a, b)=d(A, B) \text { for some } a \in A^{0}\right\} \\
& =B \cap\left\{b \in M: d(a, b) \leq d(A, B) \text { for some } a \in A^{0}\right\} \\
& =B \cap\left(\bigcup_{a \in A^{0}} B(a, d(A, B))\right)=B \cap\left(A^{0}+d(A, B)\right) .
\end{aligned}
$$

Since $A^{0}$ is admissible, then by Lemma $1.2, A^{0}+d(A, B)$ is also admissible. Thus, $B^{0}$ is admissible (note, that $B$ is admissible).

(b) Let $\left\{b_{n}\right\}$ be a sequence in $B^{0}$ such that $b_{n} \rightarrow b \in B$. Then there exists sequence $\left\{a_{n}\right\}$ in $A$ such that $d\left(a_{n}, b_{n}\right)=d(A, B)$. Since $A^{0}$ is compact, we may assume that $a_{n} \rightarrow a \in A^{0}$. Then

$$
d(a, b)=\lim _{n \rightarrow \infty} d\left(a_{n}, b_{n}\right)=d(A, B)
$$

Thus, $b \in B^{0}$. Therefore, $B^{0}$ is closed and so compact.

Theorem 2.2. Let $(M, d)$ be a hyperconvex metric space, $A \subseteq M$ and $B \subseteq M$ are admissible. Let $g: A^{0} \rightarrow A^{0}$ be a homeomorphism, and let $F: A \multimap B$ be an upper semicontinuous set-valued map with admissible values. Assume that $A^{0}$ is compact and admissible and $F(x) \cap B^{0}$ is nonempty, for each $x \in A^{0}$. Then there exists $a \in A^{0}$ such that $d(g(a), F(a))=d(A, B)$.

Proof. We use some ideas from [1, Theorem 3.2]. From [11, Proposition 2.8], $A^{0}$ is nonempty. Since $A^{0}$ and $B$ are admissible, it follows from Theorem 2.1(a) that $B^{0}$ is admissible. From Lemma 1.3, $P_{A^{0}} \in \mathbb{V}\left(B^{0}, A^{0}\right)$ (note, that $A^{0}$ is nonempty, admissible, and compact and $B^{0}$ is hyperconvex since $B^{0}$ is an admissible subset of $\left.M\right)$. Define $G: A^{0} \multimap B^{0}$ by $G(x)=F(x) \cap B^{0}$. Since $F$ is upper semicontinuous with nonempty admissible values and $B^{0}$ is admissible, then $G$ is upper semicontinuous with admissible (in particular acyclic) values. From Lemma 1.3 (see proof), $P_{A^{0}}: B^{0} \multimap A^{0}$ is upper semicontinuous with admissible (in particular acyclic) values. Since $P_{A^{0}} \circ G \in \mathbb{V}_{c}\left(A^{0}, A^{0}\right)$, it follows from Corollary 1.5 that there exists $a \in A^{0}$ such that $g(a) \in P_{A^{0}} \circ G(a)$. Thus, there exists $b \in G(a)$ such that $g(a) \in P_{A^{0}}(b)$. Hence, $g(a) \in P_{A^{0}}(b) \subseteq A^{0}$ and $b \in G(a)=F(a) \cap B^{0}$. Since $b \in B^{0}$, there exists $a_{0} \in A^{0}$ such that $d\left(a_{0}, b\right)=d(A, B)$ and hence

$$
d(A, B) \leq d(g(a), F(a)) \leq d(g(a), b)=d\left(b, A^{0}\right) \leq d\left(a_{0}, b\right)=d(A, B) .
$$

Then

$$
d(g(a), F(a))=d(A, B) .
$$


If we take $g=I_{A^{0}}$, we get the following corollary.

Corollary 2.3. Let $(M, d)$ be a hyperconvex metric space, $A \subseteq M$ and $B \subseteq M$ are admissible. Let $F: A \multimap B$ be an upper semicontinuous set-valued map with admissible values. Assume that $F(x) \cap B^{0}$ is nonempty, for each $x \in A^{0}$ and $A^{0}$ is compact and admissible. Then there exists $a \in A^{0}$ such that $d(a, F(a))=d(A, B)$.

Corollary 2.4 (see $[8$, Corollary 5.6]). Let $(M, d)$ be a hyperconvex metric space and $A \subseteq M$ nonempty, compact, and admissible. Let $F: A \multimap M$ be an upper semicontinuous set-valued map with admissible values. Assume that $F(x) \cap A$ is nonempty, for each $x \in A$. Then $F$ has a fixed point.

\section{References}

[1] M. A. Al-Thagafi and N. Shahzad, "Best proximity pairs and equilibrium pairs for Kakutani multimaps," Nonlinear Analysis: Theory, Methods E Applications, vol. 70, no. 3, pp. 1209-1216, 2009.

[2] W. K. Kim and K. H. Lee, "Corrigendum to: "Existence of best proximity pairs and equilibrium pairs"," Journal of Mathematical Analysis and Applications, vol. 329, no. 2, pp. 1482-1483, 2007.

[3] W. K. Kim and K. H. Lee, "Existence of best proximity pairs and equilibrium pairs," Journal of Mathematical Analysis and Applications, vol. 316, no. 2, pp. 433-446, 2006.

[4] S. Sadiq Basha and P. Veeramani, "Best proximity pair theorems for multifunctions with open fibres," Journal of Approximation Theory, vol. 103, no. 1, pp. 119-129, 2000.

[5] P. S. Srinivasan and P. Veeramani, "On best proximity pair theorems and fixed-point theorems," Abstract and Applied Analysis, vol. 2003, no. 1, pp. 33-47, 2003.

[6] N. Aronszajn and P. Panitchpakdi, "Extension of uniformly continuous transformations and hyperconvex metric spaces," Pacific Journal of Mathematics, vol. 6, pp. 405-439, 1956.

[7] M. Borkowski, D. Bugajewski, and H. Przybycien, "Hyperconvex spaces revisited," Bulletin of the Australian Mathematical Society, vol. 68, no. 2, pp. 191-203, 2003.

[8] J.-H. Kim and S. Park, "Comments on some fixed point theorems in hyperconvex metric spaces," Journal of Mathematical Analysis and Applications, vol. 291, no. 1, pp. 154-164, 2004.

[9] R. Sine, "Hyperconvexity and approximate fixed points," Nonlinear Analysis: Theory, Methods $\mathcal{E}$ Applications, vol. 13, no. 7, pp. 863-869, 1989.

[10] S. Park and H. Kim, "Coincidences of composites of u.s.c. maps on H-spaces and applications," Journal of the Korean Mathematical Society, vol. 32, no. 2, pp. 251-264, 1995.

[11] W. A. Kirk, S. Reich, and P. Veeramani, "Proximinal retracts and best proximity pair theorems," Numerical Functional Analysis and Optimization, vol. 24, no. 7-8, pp. 851-862, 2003. 